\title{
Editorial zum Themenschwerpunkt Arthrolysen
}

Die Arthrolyse, in offener Technik oder arthroskopisch durchgeführt, gehört seit langem zum Standard therapeutischer Möglichkeiten bei Gelenkkontrakturen und Arthrofibrosen. Das Verfahren berücksichtigt sowohl intraartikuläre Hemmnisse wie Narbenstränge, lokale Fibrosierungen, freie Gelenkkörper oder knöcherne Sperren als auch extraartikuläre Ursachen (Kapselschrumpfung, Vernarbungen, paraartikuläre Verkalkungen). Die Ergebnisse sind abhängig von Ursache und Ausmaß der Gelenksteife, der Zeitdauer der Einsteifung, einer evtl. vorausgegangenen Infektion und der Konsequenz der Nachbehandlung. Nach der Arthrolyse muss mit einer Verschlechterung des erreichten relativen Bewegungsgewinns um ca. 20\% gerechnet werden. Das Gesagte gilt für Gelenksteifen nach Verletzungen, rekonstruktiven Eingriffen und Infektionen.
Während in dieser Heftreihe das standardisierte operative Vorgehen bei Steifen verschiedener Gelenke bereits publiziert ist, gibt es Sonderindikationen, die besprochen werden müssen. Sie sind in einem Schwerpunkt zusammengefasst. Pennig et al. gehen in ihrem Beitrag auf die Wichtigkeit der Distraktion bei der Arthrolyse des Ellenbogens ein. Fuhrmann \& Wagner sehen beim lähmungsbedingten kontrakten Spitzfuß eine Indikation zur Arthrolyse in Kombination mit dem Tibialis-posterior-Transfer. Freiling \& Lobenhoffer widmen sich besonders der Streckhemmung des Kniegelenks nach Trauma oder Operation. Anagnostakos et al. schließlich konzentrieren sich auf die operative Beseitigung knöcherner Ankylosen des Hüftgelenks.

Carl Joachim Wirth 